\title{
A Cardiopulmonary Bypass Based Blood Management Strategy in Adult Cardiac Surgery
}

\author{
Ali Baran Budak, MD, ${ }^{1}$ Kevin McCusker, MD, ${ }^{2}$ Serdar Gunaydin, $\mathrm{MD}^{1}$ \\ ${ }^{1}$ Numune Training \& Research Hospital, Ankara, Turkey; ${ }^{2}$ New York Medical College, Valhalla, NY, USA
}

\section{ABSTRACT}

Background: Despite the recent introduction of a number of technical and pharmacologic blood conservation measures, bleeding and allogeneic transfusion remain persistent problems in open-heart surgical procedures. Efforts should be made to decrease or completely avoid transfusions to avoid these negative reactions.

Methods: Our coronary artery bypass grafting database was reviewed retrospectively and a total of 243 patients who underwent cardiac surgery with cardiopulmonary bypass (CPB) were studied in a 12-month period (January-December 2016) after the implementation of the new program, and compared with 275 patients of the previous 12-month period.

All the staff involved in the care of the patients were educated about the risks and benefits of blood transfusions and the new transfusion guidelines in a 45 -min training. We revised our guidelines for transfusions based on the STS. A transfusion $\log$ was created. Reduction in IV fluid volume was targeted. CPB circuitry was redesigned to achieve significantly less prime volume.

Results: The proportion of patients transfused with red blood cells was 56\% $(\mathrm{n}=154)$ in the control group and reduced by $26.8 \%$ in the study group $(29.2 \% ; 71$ patients; $P<.01)$. Blood transfusion rate $(1.7 \pm 1 / 3.05 \pm 1$ units $)$, postoperative hemorrhage $(545 \pm 50 / 775 \pm 55 \mathrm{~mL})$, respiratory support duration $(12.4 \pm 7 / 16.8 \pm 8 \mathrm{~h})$ and ICU stay $(2.2 \pm 1.1 /$ $3.5 \pm 1.2$ days) were significantly better in the blood conservation group.

Conclusion: These findings, in addition to risks and side effects of blood transfusion and the rising cost of safer blood products, justify blood conservation in adult cardiac operations.

\section{INTRODUCTION}

Despite the recent introduction of a number of technical and pharmacologic blood conservation measures, bleeding and allogeneic transfusion remain persistent problems in open-heart surgical procedures. The use of blood products carries several risks, such as immunologic sensitization, anaphylactic reaction, and disease transmission. The underlying pathophysiology has not been described entirely; however,

Received March 17, 2017; accepted April 5, 2017.

Correspondence: Serdar Gunaydin, MD, Deparment of Cardiovascular Surgery, Numune Training \& Research Hospital, Ankara, Turkey; +90-536-3896521 (e-mail: sgunaydin@isnet.net.tr). there is evidence on the activation of inflammatory genes and cytokines in circulating leukocytes with transfusion of red blood cells [Escobar 2007]. Efforts should be made to decrease or completely avoid transfusions to avoid these negative reactions.

In an effort to reduce the negative impact of $\mathrm{CPB}$ on the adult population outcome, the minimized systems were developed to significantly reduce the hemodilution of the patient and reduce the foreign surface area that comes in continuous contact with the patient circulating blood volume. Reducing the size of the perfusion apparatus has two obvious and immediate effects: first, a reduction in the contact surface area for potential blood-biomaterial contact; and second, a reduction in priming volume requirement-less hemodilution with a consequential reduction in perioperative hematocrit [Gunaydin 2009].

There is wide variation in the prevalence of perioperative transfusions in cardiac surgery. A 2013 audit of cardiac surgical units in the United Kingdom identified a threefold variation $(22-67 \%)$ in the number of patients receiving at least one unit of blood perioperatively [Murphy 2013]. Variation in blood usage and the need to balance risks and benefits have encouraged the use of pragmatic and evidence-based strategies to optimize blood component administration.

The large differences between institutions cannot be explained just by differences in patient characteristics. Most likely, institutional and individual differences in transfusion practice, guidelines, and attitudes influence the frequency and number of transfusions. The decision to transfuse is based on multiple patient factors and it is impossible to designate a single transfusion trigger. The high prevalence initiated a multifactorial blood conservation program with the intention of reducing transfusions without compromising patient safety [Arnold 2006].

The World Health Organization encourages all member states to implement Patient Blood Management programs employing multiple combined strategies to increase and preserve autologous erythrocyte volume in order to minimize unnecessary exposure to RBC transfusions [Farmer 2013].

The aim of this retrospective study was to evaluate the efficacy of a structured program to reduce transfusions and transfusion-associated complications in adult cardiac surgery.

\section{PATIENTS AND METHODS}

After institutional review board approval (14/227-2) in a tertiary cardiac center, the coronary artery bypass grafting (CABG) database was reviewed retrospectively and a total of 
Table 1. Preoperative Characteristics of the Patients

\begin{tabular}{lccc}
\hline & $\begin{array}{c}\text { Blood Management } \\
\text { Group 1 }(\mathrm{n}=243)\end{array}$ & $\begin{array}{c}\text { Control } \\
\text { Group 2 }(\mathrm{n}=275)\end{array}$ & $P$ \\
\hline Age & $69.4 \pm 10$ & $66.1 \pm 10$ & $>.05$ \\
Sex (male) & 117 & 126 & $>.05$ \\
BSA (m2) & $1.69 \pm 0.05$ & $1.73 \pm 0.05$ & $>.05$ \\
Preop EF (\%) & $0.45 \pm 0.08$ & $0.48 \pm 0.08$ & $>.05$ \\
EuroSCORE & $6.6 \pm 2.3$ & $6.9 \pm 1.5$ & $>.05$ \\
Anti-platelet therapy, $\mathrm{n}$ & 195 & 204 & $>.05$ \\
CADX1 & 17 & 14 & $>.05$ \\
CADX2 & 64 & 73 & $>.05$ \\
CADX3 & 69 & 79 & $>.05$ \\
CADX4+ & 93 & 109 & .036 \\
\hline
\end{tabular}

BSA indicates body surface area; EF, ejection fraction detected by echocar-

diography; CAD, coronary artery disease.

243 patients who underwent cardiac surgery with cardiopulmonary bypass (CPB) (Group 1: blood conservation) were studied in a 12-month period (January-December 2016) after the implementation of the new program, and compared with 275 patients (Group 2: control-no blood conservation) of the previous 12-month period.

The outcome (dependent-variable) was stated as blood transfusion. Independent variables were identified.

Exclusion criteria consisted of known coagulopathy and endocarditis. Patients on anti-platelet therapy preoperatively received the same protocol. The remainder of the patients who did not have the exclusion criteria were included in the study.

The blood conservation program was designed as follows:

Education: All the staff involved in the care of the patients, including surgeons, anesthetists, residents, OR-, ICU- and ward nurses, nurse helpers, physiotherapists and perfusionists were educated about the risks and benefits of blood transfusions and the new transfusion guidelines in a 45-minute lesson. The lesson was repeated for all new employees.

Guidelines: We revised our guidelines for transfusions based on the Society of Thoracic Surgeons Guidelines [Senay 2009]. In the institutional guidelines, the decision to transfuse red cells perioperatively should be based on clinical judgment of the patient's clinical and hemodynamic status. Generally, patients were supposed to receive red blood cells if they had a hematocrit level of $<20 \%$, mixed venous oxygen saturation $<60 \%$ and regional cerebral oxygenation $(\mathrm{rSO} 2)<50 \%$. The final decision to transfuse or not was always at the discretion of the physician responsible.

Transfusion log: A specific transfusion log was added to the patient records. In this $\log$, all transfusion episodes were registered together with time of transfusion, indication for transfusion, type of blood product, amount (units), and patient status including blood pressure, pulse, mixed venous oxygenation, hematocrit levels and the prescribing physician.
Table 2. Significant Predictors of Alllogeneic Transfusions

\begin{tabular}{lccc}
\hline Risk Factors & Odds Ratio & Confidence Interval & Multivariate $P$ \\
\hline $\begin{array}{l}\text { Lower body surface } \\
\text { area }\end{array}$ & 2.160 & $1.200-8.4$ & .0023 \\
$\begin{array}{l}\text { Lower preoperative } \\
\text { hematocrit }\end{array}$ & 2.3 & $1.100-6.500$ & .0045 \\
$\begin{array}{l}\text { Lower red blood cell } \\
\text { mass }\end{array}$ & 1.1 & $0.850-0.970$ & .076 \\
$\begin{array}{l}\text { Increased total crystal- } \\
\text { loid volume }\end{array}$ & 3.6 & $1.2-8.5$ & .041 \\
$\begin{array}{l}\text { Conventional circuit } \\
\text { size }\end{array}$ & 3.1 & $1.290-8.540$ & .047 \\
$\begin{array}{l}\text { Increased postopera- } \\
\text { tive bleeding in } 24 \mathrm{~h}\end{array}$ & 4.7 & $1.150-7.150$ & .0034
\end{tabular}

Reduction in IV fluid volume: We initiated a series of measures aimed at reducing hemodilution volume. All sources and volumes of IV fluid were obtained from the medical record.

CPB circuit design: significantly less prime volume via oxygenator with integrated arterial filter, condensed circuit, pole mounted vents, microplegia, ultrafiltration, use of cerebral oxymetry-rSO2, retrograde autologous priming, vacuum assisted venous drainage, and cell salvage of the residual blood from the circuit without treatment.

\section{Operative Technique}

As a part of the blood conservation program, the bypass circuit was aseptically assembled using a low-prime integrated oxygenator and reservoir (Fx15, Terumo Medical Corporation, Ann Arbor, MI, USA) in Group 1 and a Terumo System 1 pump console. All of the roller heads of the pump were remotely mounted to minimize tubing length and circuit surface area. Isothermic blood cardioplegia was administered directly into the aorta at the surgical field using premixed syringes of a 1:4 hyperkalemic solution (1 part blood: 4 parts crystalloid) (Pharmedium, Portland, OR, USA). The static prime volume for the circuit was approximately $250 \mathrm{~mL}$.

As part of our previous routine, the control group consisted of a conventional oxygenator and reservoir (Sorin, Arvada, $\mathrm{CO}, \mathrm{USA}$ ) that has a static priming volume of $450 \mathrm{~mL}$.

Anticoagulation status was monitored using the Medtronic HMS+ (Medtronic, Minneapolis, MN, USA) system. Samples of blood for measuring activated clotting times (ACT) and heparin concentrations were drawn every 30 minutes on CPB. Adequate anticoagulation was defined as ACT greater than 500 seconds and heparin concentration assays greater than or equal to $3.0 \mathrm{mg} / \mathrm{kg}$, as measured by the $\mathrm{HMS}+$ device. Midazolam, sevoflurane, fentanyl, and norcuronium were used as anesthetic agents.

After median sternotomy, the patient was systemically anticoagulated using 339 units $/ \mathrm{kg}$ of intravenous heparin. The pulse pressure was verified and retrograde autologous priming (RAP) was initiated. 
Table 3. Perioperative Outcome

\begin{tabular}{lccc}
\hline & $\begin{array}{c}\text { Blood Conservation } \\
\text { Group 1 }\end{array}$ & $\begin{array}{c}\text { Control } \\
\text { Group 2 }\end{array}$ & $P$ \\
\hline Duration of CPB, min & $90.4 \pm 46$ & $92 \pm 42$ & .98 \\
Duration of x-clamp, min & $54.4 \pm 16$ & $62.2 \pm 17$ & .94 \\
t-intub, h & $12.4 \pm 7^{*}$ & $16.8 \pm 8$ & .044 \\
Postop hemorrhage, mL & $545 \pm 50^{*}$ & $775 \pm 55$ & .046 \\
Blood transfusion, unit & $1.7 \pm 1^{*}$ & $3.05 \pm 1$ & .015 \\
Inotropic support, n & $75(37.8 \%)^{*}$ & $115(56 \%)$ & .017 \\
ICU stay, day & $2.2 \pm 1.1^{*}$ & $3.5 \pm 1.2$ & .045 \\
Hospital stay, day & $6.4 \pm 3$ & $7.5 \pm 4$ & .065 \\
Mortality, n & - & 2 & .24
\end{tabular}

Vacuum-assisted venous drainage was utilized to allow for the use of the smaller venous cannula and venous circuit. Upon initiation of bypass, crystalloid in the venous line was diverted into a collection bag and discarded. Full flow to the patient was achieved with no hemodynamic instability within five minutes of aortic cannulation.

CPB flow was maintained at $125-150 \mathrm{~mL} / \mathrm{kg} /$ minute. Moderate hypothermia $\left(28-34^{\circ} \mathrm{C}\right)$ and alpha stat blood gas management were used.

After the surgical procedure, the heart was aggressively deaired. The aortic cross-clamp was removed and the patient was systemically rewarmed to normothermia. A second blood gas was drawn to ensure proper electrolyte balance prior to termination of CPB support. The resultant hematocrit was around $20 \%$. Continuous ultrafiltration was performed while the patient was on CPB. Circuit integrity was maintained until the patient was transported to the pediatric intensive care unit.

\section{Perioperative Follow-up}

For each patient, the following factors were evaluated before discharge and documented:

Hemodynamic parameters, perfusion and cross-clamp duration, intubation period, postoperative hemorrhage, use of blood (as units) during the hospital stay, use of inotropic support, complications and infection, the duration of ICU stay and hospital stay, perioperative mortality. A comparison between groups was performed retrospectively.

\section{Blood Samples and Assays}

Complete blood count (hemoglobin, hematocrit, erythrocyte, white blood cell [WBC] and platelet counts) was recorded. Results of standard blood and urine chemistry were documented.

\section{Statistical Analysis}

Data was expressed as the mean \pm standard error of the mean. Analysis of variance for comparison of parameters with normal distribution, and Mann Whitney $U$ test for comparison of parameters without normal distribution were used. Univariate analysis was performed on a large number of preoperative and intraoperative variables in our database to identify those variables that might be associated with allogeneic blood. They were entered into a stepwise multiple logistic regression analysis to select the ones that were significant independent predictors of the need for transfusion. Data were analyzed using SPSS program.

\section{RESULTS}

Preoperative evaluation of groups is summarized in Table 1. The two groups were generally comparable preoperatively.

Preoperative physical and laboratory evaluation revealed no difference between patient groups. A list of variables that were screened for possible association with transfusion (dependent variable: transfused allogeneic blood) and significant predictors of allogeneic transfusions are listed in Table 2.

The proportion of patients transfused with red blood cells was $56 \%(\mathrm{n}=154)$ in the control group and reduced by $26.8 \%$ in the study group $(29.2 \% ; 71$ patients; $P<.01)$.

Perioperative outcome is listed in Table 3.

\section{DISCUSSION}

Our main motive for organizing a structured patient blood management program was to document that the proportion of patients transfused with red blood cells was $56 \%$. This was not acceptable. All staff (surgeon, anesthesiologists, nurses, and perfusionists) remained the same. Our target was to diminish transfusion without compromising patient safety.

We published several CPB-related blood preservation studies before and demonstrated the positive impact of each strategy separately [Gourlay 2008; Gunaydin 2011; McCusker 2006; Senay 2009]. In this project, we implemented whatever we thought we could benefit from. Our main prospect was to employ novel $\mathrm{CPB}$ techniques that would result in less hemodilution.

CPB-related technologies for targeting blood preservation were all confirmed on an evidence-based level in recent guidelines [Ferraris 2011]: Condensed CPB circuit as Class I-A; microplegia as Class IIb-B; vacuum-assisted venous drainage as Class IIb-C; retrograde autologous priming as Class IIb-B and ultrafiltration as Class IIa. A multi-disciplinary blood conservation program was also cited as Class IIa evidence.

One of the greatest benefits of the low prime condensed circuits is in the reduction of CPB prime by eliminating an external arterial line filter and the associated filter bypass loop and extra tubing needed to encompass the use of an arterial line filter. The elimination of an external arterial filter has not only reduced the overall $\mathrm{CPB}$ surface area but has also allowed us to decrease our overall prime volumes. This has been accomplished through a reduction in static prime volume and also by allowing placement of the oxygenator closer to the patient.

In our series, for the blood conservation group, the proportion of patients transfused with red blood cells was $56 \%$ $(n=154)$ in the control group and reduced by $26.8 \%$ in the 
study group $(29.2 \% ; 71$ patients, $P<.01)$. Blood transfusion rate $(1.7 \pm 1 / 3.05 \pm 1$ units), postoperative hemorrhage $(545 \pm$ $50 / 775 \pm 55 \mathrm{~mL})$, respiratory support duration $(12.4 \pm 7 / 16.8$ $\pm 8 \mathrm{~h})$, and ICU stay $(2.2 \pm 1.1 / 3.5 \pm 1.2$ days $)$ were significantly better in the blood conservation group.

We studied thorough variables related to the preoperative and intraoperative period and demonstrated that the most significant risk factors for transfusion were body surface area (OR: 2.16), preoperative hematocrit (OR: 2.3), red blood cell mass (OR: 1.1), total crystalloid volume (OR: 3.6), conventional circuit (OR: 3.1), and postoperative bleeding in $24 \mathrm{~h}$ (OR: 4.7). The results of the present study demonstrate that a structured blood conservation program reduces transfusion prevalence and this can be achieved without compromising patient safety.

Several public-based blood management initiatives are in progress to optimize utilization of RBC concentrates [Menkis 2012]. However, none of these initiatives are designed to provide scientific data in terms of safety issues. Thus, a large study with robust and relevant clinical endpoints is required. The objective of this study is to demonstrate that the implementation of a patient blood management program is safe and achievable.

Our study is a retrospective study and this is one of the main limitations of the design. We accept this data as preliminary to build a strategy and started a newer protocol and assessment based on current outcomes obtained from retrospective evaluation and long-term clinical outcome. We accept that using historic data as a control group in such a comparative study has less power. But we made every effort to match both databases to make a comparison as much as is logical.

In order to continue to improve surgical outcomes, we must continue to ask the correct questions and attempt to answer them with the appropriate studies. Our patient population is small. That may be a reason not to get clear significant differences in most of the clinical parameters.

Our results confirm that transfusion of red blood cells during $\mathrm{CPB}$ may cause an undesirable clinical outcome. It is obvious that blood management with multiple strategies is valid for an adult population. Hemodilution becomes more important in lower body surface area. We believe novel CPB technologies may positively contribute to blood management strategies regarding adult patients undergoing open-heart surgery.

\section{REFERENCES}

Arnold DM, Fergusson DA, Chan AK, et al. 2006. Avoiding transfusions in children undergoing cardiac surgery: a meta- analysis of randomized trials of aprotinin. Anesth Analg 102:731-7.

Escobar GA, Cheng AM, Moore EE, et al. 2007. Stored packed red blood cell transfusion upregulates inflammatory gene expression in circulating leukocytes. Ann Surg 246:129-34.

Farmer SL, Towler SC, Leahy MF, Hofmann A. 2013. Drivers for change: Western Australia Patient Blood Management Program (WA PBMP), World Health Assembly (WHA) and Advisory Committee on Blood Safety and Availability (ACBSA). Best Pract Res Clin Anaesthesiol 27:43-58.

Ferraris VA, Brown JR, Despotis GJ, et al. 2011. 2011 update to the Society of Thoracic Surgeons and the Society of Cardiovascular Anesthesiologists blood conservation clinical practice guidelines. Ann Thorac Surg 91:944-82.

Gourlay T, Olivencia-Yurvati AH, Gunaydin S. 2008. STS blood conservation guidelines: the role of leukocyte filtration. Ann Thorac Surg 85:1138-9.

Gunaydin S, Sari T, McCusker K, Schonrock U, Zorlutuna Y. 2009. Clinical evaluation of minimized extracorporeal circulation in high-risk coronary revascularization: impact on air handling, inflammation, hemodilution and myocardial function. Perfusion 24:153-62.

Gunaydin S, McCusker K, Vijay V. 2011. Perioperative blood conservation strategies in pediatric patients undergoing open-heart surgery: impact of non-autologous blood transfusion and surface-coated extracorporeal circuits. Perfusion 26:199-205.

McCusker K, Chalafant A, De Foe G, Gunaydin S, Vijay V. 2006. Influence of hematocrit and pump prime on cerebral oxygen saturation in on-pump coronary revascularization. Perfusion 21:149-55.

Menkis AH, Martin J, Cheng DC, et al. 2012. Drug, devices, technologies, and techniques for blood management in minimally invasive and conventional cardiothoracic surgery: A consensus statement from the International Society for Minimally Invasive Cardiothoracic Surgery (ISMICS) 2011. Innovations (Phila) 7:229-41.

Murphy MF, Murphy GJ. 2013. 2011 Audit of Blood Transfusion in Cardiac Surgery. 2013. Available at: http://hospital.blood.co.uk/ media/26859/nca2011_use_of_blood_in_adult_cardiac_surgery_report. pdf. Accessed February 24, 2015.

Senay S, Toraman F, Gunaydin S, Kilercik M, Karabulut H, Alhan C. 2009. The impact of allogenic red cell transfusion and coated bypass circuit on the inflammatory response during cardiopulmonary bypass: a randomized study. Interact CardioVasc Thorac Surg 8:93-9. 